\title{
CONSERVACIÓN COMUNITARIA DE LA QUÍNOA EN EL NORTE DE NEUQUÉN. ARGENTINA
}

\author{
Barrionuevo, M. E. ${ }^{1}$; Mogni, A. J. ${ }^{1}$; VÁZquez, A. ${ }^{3}$ \& Navarrete, L. ${ }^{1}$
}

\begin{abstract}
RESUMEN
Tradicionalmente agricultores familiares de Neuquén (Argentina) cultivan quínoa (Chenopodium quinoa Willd.), el estudio pone en valor un material genético de quínoa en Varvarco. Se identificaron y entrevistaron a las familias guardianas de las semillas de quínoa; se realizaron observaciones del cultivo in situ y se validó la información en talleres con la comunidad. Los resultados mostraron que la quínoa forma parte del patrimonio histórico y cultural de Varvarco ya que contribuyó a la seguridad alimentaria en épocas de crisis económicas producto de distintos eventos históricos que afectaron económica y culturalmente al norte neuquino. Lleva al menos 90 años en la zona, el ciclo de cultivo es de noviembre a marzo, con características fenotípicas y de manejo similares a los materiales del Centro de Chile. El estudio mejora el conocimiento el comportamiento de la quínoa local para adaptar los calendarios, formas de manejo del cultivo, suelo y sanidad en otros agroecosistemas.
\end{abstract}

Palabras clave: Chenopodium quinoa Willd., criollos, agricultura familiar.

1.- INTA IPAF Región Patagonia, Autovía 22 s/n, Colonia San Francisco. (8316) Plottier. Neuquén. Argentina. Email: myrianbarrionuevo@gmail.com

2.- Agencia de Extensión Rural Chos Malal, Mitre 36. (8353) Chos Malal, Neuquén. Argentina.

3.- Dirección Regional Norte de Producción, Ministerio de Producción de Industria. Lamadrid 153. (8353)

Chos Malal, Neuquén. Argentina.

4.- Becaria INTA AUDEAS CONADEV Proyecto CIAC - 940167. Facultad de Ciencias Agrarias, Universidad Nacional del Comahue. Ruta Nacional 151 km.12,5. (8303)Cinco Saltos, Río Negro. Argentina. Manuscrito recibido el 28 de abril de 2020 y aceptado para su publicación el 20 de julio de 2020.

Barrionuevo, M.E.; Mogni, A.J.; Vazquez, A.; Navarrete; L. Conservación comunitaria de la quínoa (Chenopodiun quínoa Willd.) en el norte de Neuquén. Argentina. FAVE - Ciencias Agrarias 20 (1): 361-377.

CC BY-NC-SA 4.0 


\begin{abstract}
Community conservation of quinoa in northern Neuquén.

This study aimed to highlight the local variety of Quinoa from Varvarco. The research took into account the guardian families of quinoa seeds. The team identified and interviewed family farmers; the crop was observed in situ and the information was validated in workshops with the Varvarco community. The results showed that this quinoa is part of Varvarco's historical and cultural heritage, it contributed to food security in times of economic crisis because of different historical events that affect the economy and culture of the north of Neuquén. This quinoa has been in this area for at least 90 years. The cultivation cycle is from November to March, its phenotypic characteristics and management are similar to Central Chile varieties. The study improves the knowledge of quinoa behavior to adapt the calendars, forms of crop management, soil and plant protection in other agroecosystems.
\end{abstract}

Key words: Chenopodium quinoa Willd, creole, family agriculture.

\section{INTRODUCCIÓN}

La quínoa (Chenopodium quinoa Willd.) es un cultivo que crece en ambientes heterogéneos a lo largo de la región andina, desde los $2^{\circ}$ de latitud Norte en Colombia hasta los $40^{\circ}$ de latitud Sur en Chile (Wilson 1990) y desde las regiones costeras del Pacífico a los altiplanos por encima de los 4000m.s.n.m.. Adaptada a condiciones de humedad relativas entre 40 y $88 \%$ y temperaturas que van de $-4^{\circ} \mathrm{C}$ a $38^{\circ} \mathrm{C}$. Se trata de una especie autógama nativa de América del Sur de amplia variabilidad genética (Bioversity International 2013, Hunziker 1943). Esta variabilidad y distribución geográfica resultan de las interacciones entre los productores y el ambiente de acuerdo al uso, formas de consumo, adquisición y circulación que definen sus características particulares según el contexto (Costa Tártara et al. 2012).
Su importancia como alimento radica en la calidad nutritiva de sus granos representada por su composición de aminoácidos esenciales tanto en calidad como en cantidad. Esta característica motiva su estudio, conservación y mejoramiento genético en diversas partes del mundo.

En Argentina el cultivo de quínoa tuvo una extensa distribución en el pasado prehispánico situándose desde el extremo norte en la provincia de Jujuy, pasando por las provincias de Salta, Catamarca, Tucumán, San Juan y Mendoza, con extensiones que llegaron a Santa Fe y Córdoba y hacia el sur en las provincias patagónicas de Neuquén, Río Negro y Chubut (Parodi 1998, Recalde 2007, Daza et al. 2015, Maloberti et al. 2016).

En la actualidad, el cultivo comercial y familiar de quínoa en Argentina se sitúa principalmente en las provincias del Noroeste, en tanto que en otras regiones como la patagónica se limita a la producción familiar para el autoconsumo (Bertero 2001, Barrionuevo et al. 2019). 
En la provincia del Neuquén el cultivo de quínoa subsiste en comunidades mapuche y criollas (Azar 2002) y las colecciones en los bancos de germoplasma dan cuenta de la distribución a lo largo de la cordillera patagónica (Knunzen 2000). Así mismo, los testimonios de los pobladores y técnicos del sistema de extensión confirman la presencia del cultivo desde los parajes Coyuco y Cochico en el norte a los lagos del sur de la provincia (Lator et al. 2015). Por otra parte, en el año 2004 se presentó en la Secretaría de Planificación y Desarrollo de Neuquén (COPADE) una propuesta llamada "Quínoa cultivo no tradicional... un camino hacia la sustentabilidad de las pequeñas comunidades”, cuyo objetivo era realizar parcelas experimentales de quínoa en distintos lugares de la provincia y se probaron variedades comerciales de quínoa en el vivero de Huinganco (Aucar 2004, LM 2013). Si bien estos trabajos no tuvieron continuidad, el personal del vivero y algunos productores familiarizados con el cultivo de quínoa multiplicaron las variedades comerciales y materiales locales. Las semillas obtenidas se compartieron a otros agricultores y los materiales circularon por la zona, llegando a las ferias de intercambio de semillas además de cultivarse en distintos puntos de la provincia.

Las ferias de semillas constituyen un espacio social y cultural donde se favorece el intercambio de materiales genéticos y conocimientos (Gallardo 2019). Son parte del sistema informal de semillas y permiten poner en valor los materiales genéticos locales. Según el registro de la feria de la Zona Centro, en el año 2012 una de las participantes procedente de Varvarco llevó semillas de quínoa para intercambiar, estableciéndose de esta forma los primeros contactos con las familias productoras de quínoa.
Con el propósito de conocer el estado actual de la agrobiodiversidad en el norte neuquino se realizaron diversas actividades en el marco del proyecto INTA AUDEAS CONADEV denominado "Caracterización y conservación de recursos fitogenéticos locales de la Agricultura Familiar en la región de Cuyo y Norpatagonia”. Este artículo es parte de un estudio más amplio y tiene por objetivo poner en valor de manera colectiva la quínoa local del norte neuquino, situado en la zona rural del ejido municipal de Varvarco. Para ello se identificaron y entrevistaron a las familias guardianas de las semillas de quínoa; se realizaron observaciones del cultivo in situ para conocer el ciclo y características generales del material; se sistematizó la información recolectada de diversas fuentes y se validaron los resultados con la comunidad de Varvarco.

\section{MATERIALES Y MÉTODOS}

El estudio se desarrolló entre octubre de 2017 y diciembre de 2018 en la localidad y área rural de Varvarco $\left(36^{\circ} 48^{\prime} \mathrm{S}, 72^{\circ} 42^{\prime} \mathrm{O}\right)$ provincia del Neuquén, Patagonia Argentina (Figura 1). La Comisión de Fomento de Varvarco de 585 habitantes (INDEC 2010) se encuentra ubicada a $1250 \mathrm{~m}$. s.n.m. La temperatura media anual es de $10.5^{\circ} \mathrm{C}$, con veranos cálidos de temperaturas medias mensuales de $13.6^{\circ}, 16.4^{\circ}, 17,6^{\circ}$ y $16.8^{\circ} \mathrm{C}$ registradas en noviembre, diciembre, enero y febrero respectivamente. La precipitación anual de $680 \mathrm{~mm}$ se concentra en los meses de mayo, junio y julio en forma de lluvia y nieve. En estas condiciones la vegetación natural es de estepa herbácea y estepa arbustiva (Figura 2). Debido a estas características es posible el desarrollo de cultivos de 


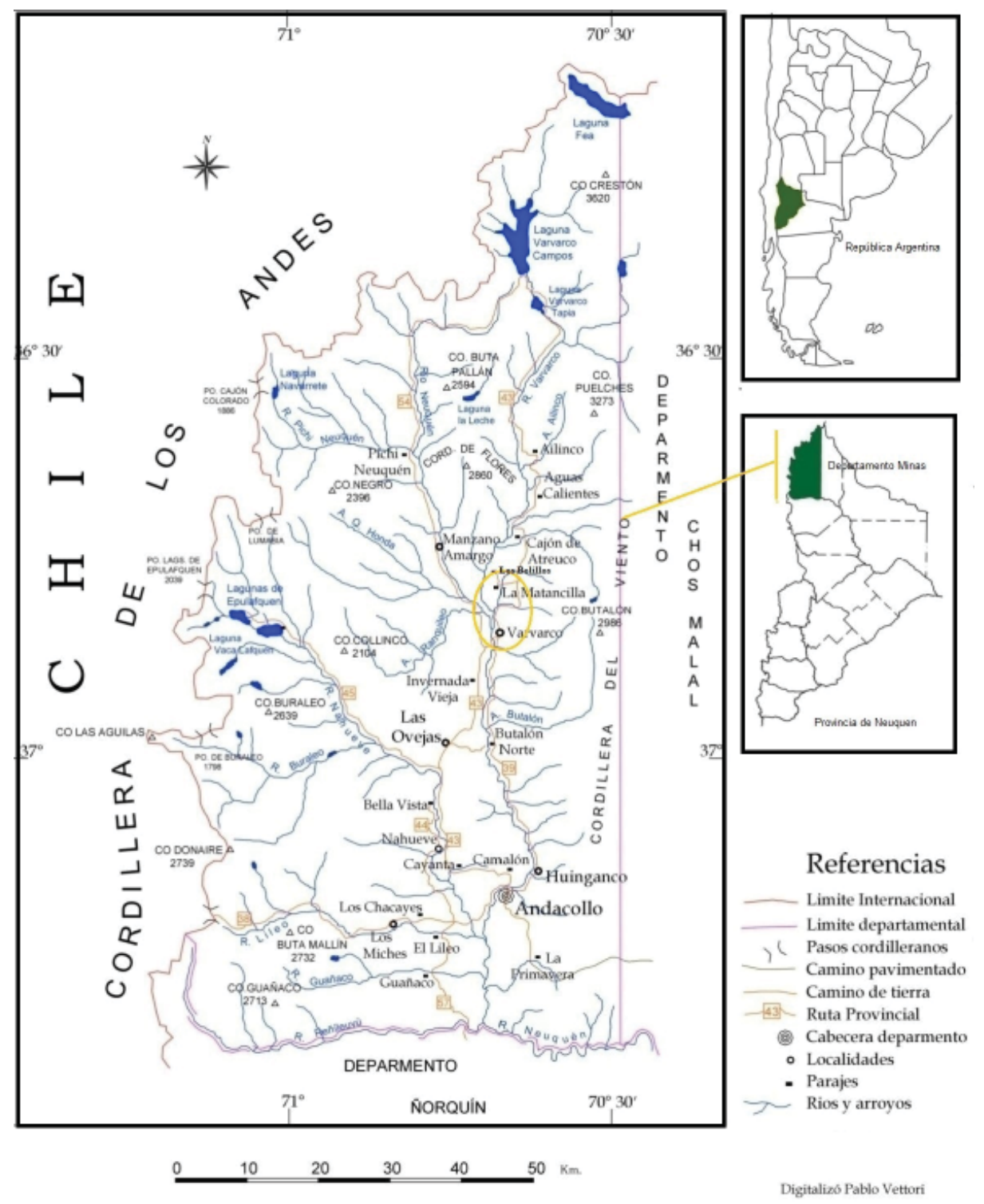

Figura 1. Mapa del departamento Minas, provincia de Neuquén. Argentina.

Fuente: Tomado de Bertani 2011.

Figure 1. Map of the Minas department, Neuquén province. Argentina. 


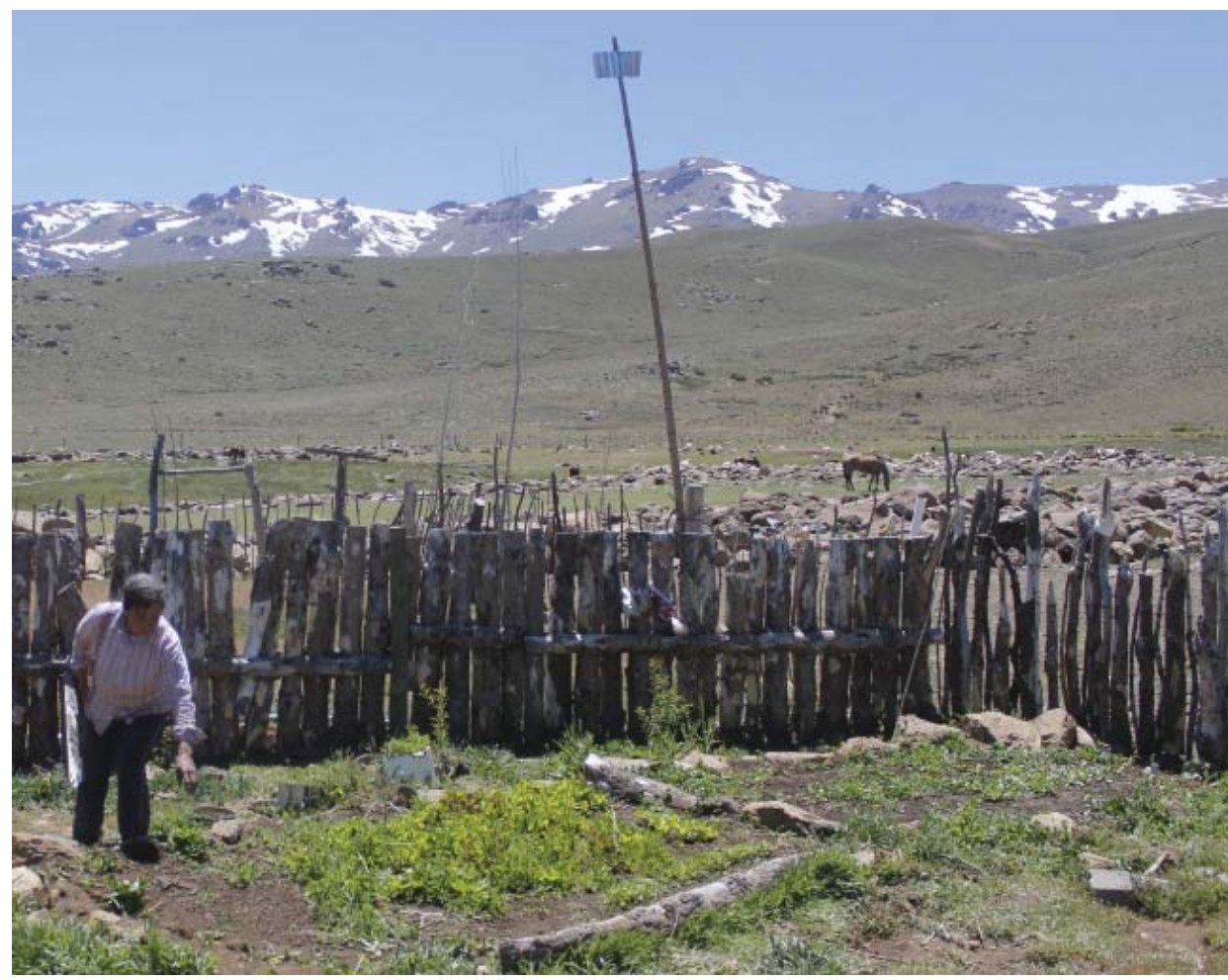

Figura 2. Paisaje del área rural de Varvarco, puesto El Auque.

Figure 2. Landscape of the rural area of Varvarco, El Auque place.

diferentes especies de cereales y hortalizas al aire libre con aplicación de riego ya que las precipitaciones estivales se encuentran por debajo de sus requerimientos (Figura 3). El agua para consumo humano y productivo proviene de arroyos y vertientes de la cuenca inferior del río Varvarco.

El área de estudio se definió con los técnicos del sistema provincial y nacional de extensión y se delimitó tomando en consi- deración los datos de pasaporte y número de accesiones del norte neuquino de las colecciones de dos bancos de la red de Bancos de Germoplasma del Instituto Nacional de Tecnología Agropecuaria (INTA) en los años 1997 y 2007.

La metodología utilizada fue cualitativa y las técnicas de recolección de la información fueron la observación participante, las entrevistas semiestructuradas y talleres con la comunidad. 


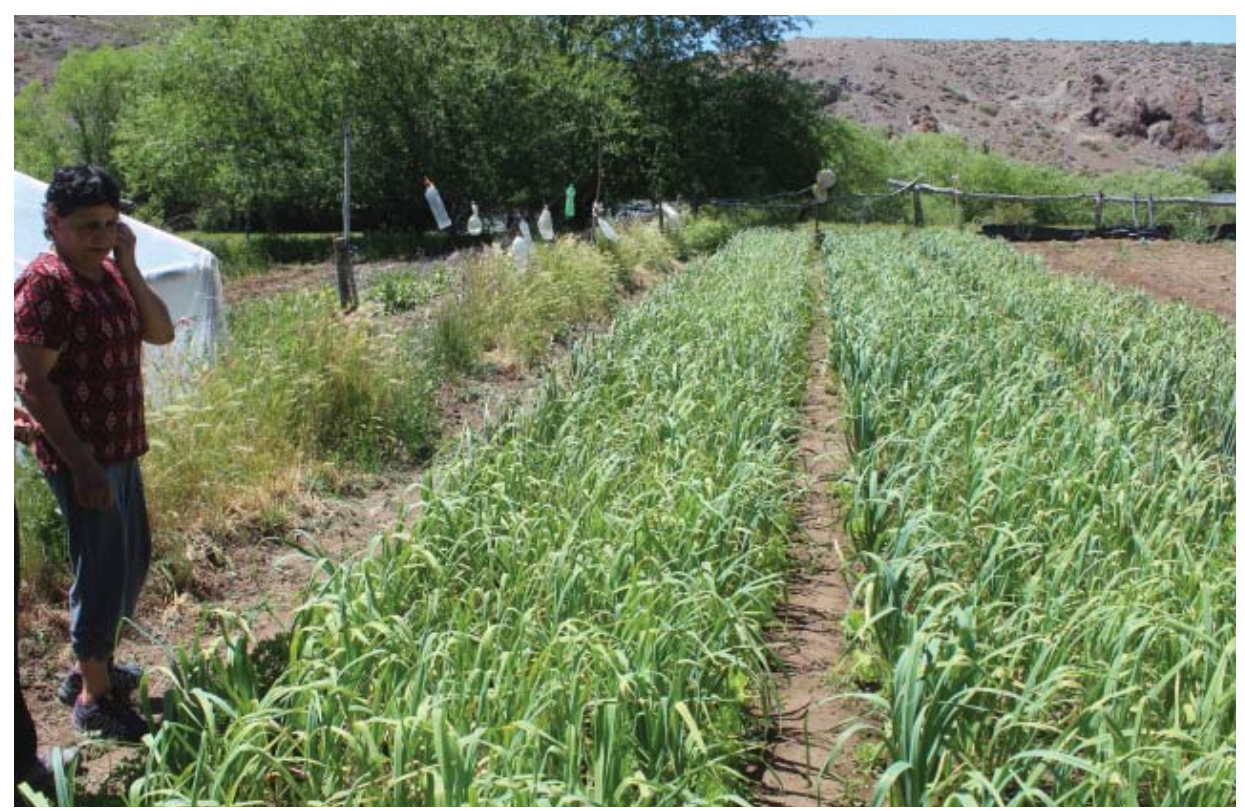

Figura 3. Huerta en el área rural de Varvarco

Figure 3. Orchard in the rural area of Varvarco

La entrevista semiestructurada consideró aspectos productivos y culturales según descriptores para quínoa de Bioversity International (2013), la que se completó con preguntas específicas sobre el origen de los aprendizajes sobre multiplicación, cultivo, usos y formas de consumo de las semillas a la familia productora. Las entrevistas fueron grabadas y sistematizadas utilizando el programa de hoja de cálculo Microsoft Excel 2013. También se realizaron registros fotográficos de las visitas a los predios para el reconocimiento de las fases fenológicas del cultivo.

Se entrevistaron 8 productores de quínoa local, los más antiguos residentes en la zona de estudio, con conocimientos de producción y multiplicación de semillas y que no cultivaran materiales exóticos de quínoa en el mismo sitio.
Para el trabajo con la comunidad de Varvarco se utilizó la metodología de taller, la planificación estuvo a cargo del equipo técnico con la colaboración del personal de la Comisión de fomento para la convocatoria y la preparación del lugar. Se realizaron 2 talleres en el año 2018 en los meses de mayo y diciembre respectivamente.

El taller de mayo tuvo como objetivo reflexionar sobre la importancia de la conservación local de quínoa y validar la información sistematizada de las entrevistas. En la apertura del taller, se presentaron los objetivos y actividades desarrolladas en la comunidad en relación al cultivo de quínoa resumidas en una línea de tiempo con el listado de personas entrevistadas. En la segunda parte se motivó la discusión e intercambio entre los asistentes utilizando para ello un esquema del cultivo de quínoa a largo del 
año, ubicando en la parte superior de una línea las etapas fenológicas y en la parte inferior las actividades culturales desarrolladas en cada etapa del cultivo con los meses del año en base a los datos provistos por los 8 productores entrevistados. Para el cierre del taller, se presentaron y degustaron productos elaborados con quínoa disponible en el mercado de la ciudad de Neuquén (harinas, galletas, barras de cereal, mezcla de semillas, entre otros). La evaluación se realizó de manera oral y se recogieron propuestas para el taller de diciembre. Como parte de la sistematización de la actividad, que tuvo una duración de 2horas y 15 minutos, se tomaron fotografías y se registraron los aportes de los participantes en papel afiche.

El segundo taller se realizó la primera quincena de diciembre de 2018 y tuvo como objetivo promover la importancia de la conservación de los recursos genéticos locales en relación al patrimonio cultural de los pueblos. En el primer momento del taller, 3 integrantes del Grupo de Promotores Culturales del norte neuquino presentaron la experiencia de rescate y puesta en valor de las tradiciones gastronómicas de esta parte de la provincia de Neuquén. Luego el equipo expuso los resultados del trabajo realizado con la comunidad en torno a la quínoa de Varvarco sintetizados en una ficha. El documento en formato papel, que se entregó a los asistentes y a la Comisión de Fomento de Varvarco, describe el ciclo de cultivo de la quínoa, principales usos y características del grano. Para el cierre una profesora de cocina tradicional presentó tres recetas a base de quínoa y se degustaron otros platos elaborados por los participantes. La evaluación consistió en responder a la pregunta: ¿Por qué es importante conservar, proteger y cultivar la quínoa en
Varvarco? El final de esta etapa dejó planteadas actividades que se continuaron por el equipo de extensionistas y que no fueron objeto del presente estudio.

Los nombres de los productores del contexto de estudio se identificaron con iniciales en mayúsculas seguidos del año de la entrevista. Conceptos y términos locales usados en el texto se escribieron entre comillas simples y las frases textuales de entrevistados y participantes de los talleres se transcribieron entre comillas dobles.

La búsqueda de información tuvo en cuenta la bibliografía específica, los registros de semillas de la feria de semillas de Zapala, notas de los cuadernos de campo de los extensionistas del programa Pro Huerta, recetarios de comidas típicas de la zona además de noticias publicadas en los periódicos locales referidas a la producción de quínoa.

\section{RESULTADOS}

En la actualidad, los agricultores familiares entrevistados tienen 58 años edad promedio, 4 de ellos, residen en Varvarco son empleados en diferentes reparticiones del estado y cultivan huertas de traspatio para el autoconsumo; los otros 4 permanecen en el área rural donde se dedican a la cría de animales y tienen pequeñas parcelas en el campo de invernada.

Los 8 productores entrevistados conocen el cultivo de quínoa desde pequeños al que denominan ‘quingua' y lo distinguen de la 'quingüilla' (Chenopodium album L.), una especie arvense con semillas negras muy pequeñas a la que consideran maleza y no tiene aprovechamiento alimenticio o medicinal como se observa en otros lugares de la 
provincia (Ladio 2005). De acuerdo al origen de las semillas, las familias denominan 'heredadas' a las que vienen de generación en generación y el movimiento es de padres a hijos o de abuelos a nietos.

Los entrevistados reconocieron a dos personas como los guardianes de semillas e impulsores del cultivo local de quínoa en Varvarco y alrededores, identificados para este estudio como GV (empleado público de 59 años) y HV (jubilado de 60 años). GV y HV estiman que sus familiares trajeron las semillas de quínoa de Chile; GV las heredó de sus abuelos chilenos, mientras que $\mathrm{HV}$ de su madre. En general, y en la medida de lo posible, se sembraba la semilla propia recurriéndose a la de otros vecinos en caso de pérdida total por incendio o mala conservación, comentó ALH productora de 61 años de edad. En la entrevista realizada a AG en noviembre de 2017, la productora de 73 años recordó que cuando era chica, intercambiaban grasa, 'charqui' (carne salada y deshidratada) por semillas y azúcar en Chile.

En los últimos años además de donar a los bancos de germoplasma, los agricultores manifestaron que compartieron las semillas por fuera del círculo familiar y local. Tal es el caso de FH, portera de la escuela de 60 años de edad que llevó semillas a la escuela primaria de Varvarco donde trabaja; HV participó activamente en las ferias de semillas de la zona Norte donde impartió charlas en talleres y GV colaboró entregando a varias personas de la comunidad que mostraron interés en sembrar. En relación con esto último, la circulación informal de semillas amplió el área de distribución de la quínoa de Varvarco hacia otros pueblos y parajes.
Los agricultores de Varvarco explicaron que para la siembra, que se inicia en octubre, el suelo debe estar bien preparado con abono de corral viejo y seco. Antes se utilizaban bueyes para la labranza, en cambio hoy se hace a mano porque se siembra poca cantidad. Una vez pasado el peligro de heladas se procede a la siembra que por lo general es en noviembre y se cosecha en marzo. En casos excepcionales de siembras tempranas de octubre la cosecha se realiza en febrero, y en las siembras tardías de fin de noviembre en abril. "La siembra debe ser rala, al voleo y luego se pasan ramas para tapar la semilla” comentó ALH (2017). El cultivo de quínoa se asocia generalmente con maíz, donde el maíz va en línea y la quínoa al voleo "[...] también puede entreverarse en la 'chacra' con maíz y poroto donde se siembra la quínoa al voleo” explicó AG (2018). En esta zona el término 'chacra' hace referencia a la parte de la huerta donde se cultivan de manera asociada maíz y poroto con zapallos, papas o quínoa.

Según el relato de HV "los animales no la comen, si los pájaros" y esto sucede los primeros 15 días cuando las plantas son pequeñas. En marzo, "cuando la hoja esta amarilla" se cortan los tallos para secar por unas 3 semanas, "cuando es poca cantidad con una semana alcanza” afirmó FH (2018). El secado de los granos se realiza con poco sol y se guardan por la noche, pasado el tiempo de secado los productores golpean con palos las panojas sobre una lona para desprender los granos y luego los limpian aventándolos antes de almacenarlos. Otra forma muy difundida consiste en limpiar los granos al momento de consumirlos, frotándolos con las manos. 
Los granos para semilla se apartan limpios y se recolectan en "bolsa de tela, frasco de vidrio o cántaro de greda" explicó HV (2018), y la cantidad puede ir desde unos cuantos puñados a 1 kilo de semillas. Algunos de los entrevistados siembran exclusivamente para conservar semillas.

La preparación de los granos para el consumo requiere quitar las saponinas que le confieren sabor amargo, para ello es necesario lavarlos con abundante agua antes de cocinar. Según AG "se lava con nueve aguas y se refriega bien porque es amarga”. El tiempo de cocción es de alrededor de 20 minutos, y una vez cocida se utiliza para elaboraciones dulces o saladas. Otra forma de aprovechamiento de acuerdo al relato de GV es tostar y moler los granos hasta lograr una harina que se emplea en otras preparaciones con leche, agua u otra bebida fría o caliente.

En las visitas prediales se observó que actualmente en Varvarco se siembra muy poca cantidad, algunas líneas de la huerta familiar o en pequeñas parcelas que no al- canzan a $0,25 h a$. Se riega por manto, principalmente los meses de diciembre y enero cuando la demanda hídrica es mayor y el raleo de plantas se realiza al mes de la siembra generalmente en diciembre. La mayoría de los productores reconoció que la falta de agua y la escasa disponibilidad de mano de obra para las tareas de campo son los factores decisivos para la continuidad del cultivo de quínoa y otras especies hortícolas en la zona.

En noviembre de 2018, 3 de los entrevistados no sembraron debido a la sequía y una de las productoras reconoció que desde hace 4 años no siembra quínoa en su terreno porque es mucho trabajo para ella.

\section{Algunas características de la quínoa de Varvarco}

La planta de quínoa de Varvarco es de color verde claro, ceniciento y el tipo de crecimiento es herbáceo y habito ramificado, con las ramas primarias que salen oblicuamente al tallo principal (Figura 4). Es

Figura 4. Planta de quínoa. Figure 4. Quinoa plant.

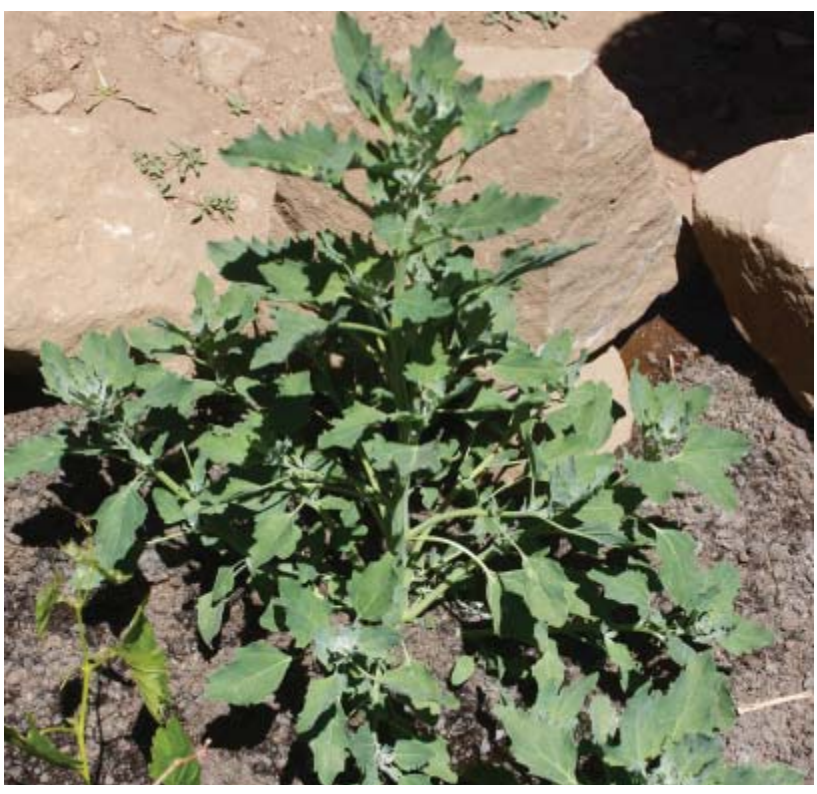




\section{E. Barrionuevo et al.}

de hojas triangulares y bordes aserrados de 5). Por su parte, la panoja es de color verde color verde y su altura varía de acuerdo a la en floración y marrón claro en la madurez calidad del suelo y la densidad de siembra. fisiológica, de forma intermedia; y los graEn huertas familiares visitadas alcanza una nos son de color crema y forma cilíndrica altura de aproximadamente 1,5m (Figura (Figura 6).

Figura 5. Inflorescencia de quínoa cultivada en Varvarco

Figure 5. Inflorescence of quinoa grown in Varvarco

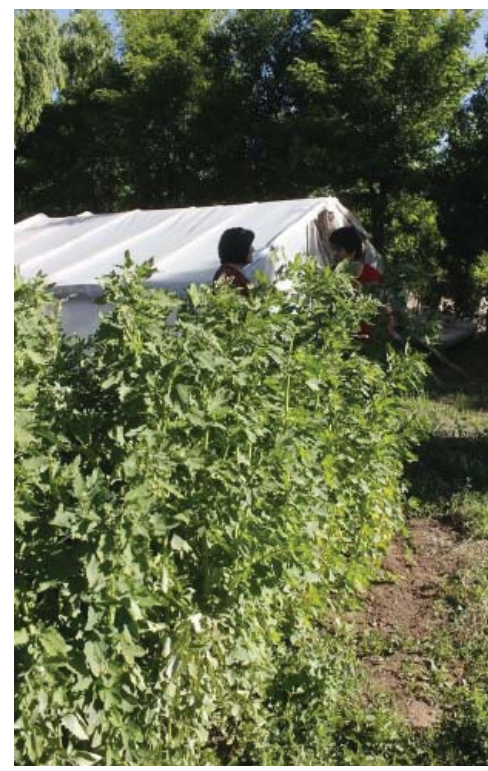

Figura 6. Cultivo de quínoa en huerta urbana de Varvarco, diciembre de 2018

Figure 6. Quinoa cultivation in the urban garden of Varvarco, December 2018.

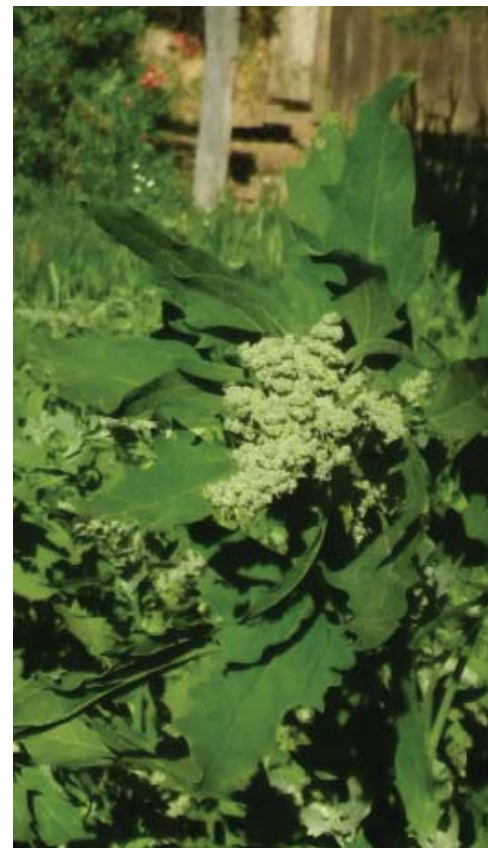


En Varvarco la emergencia ocurre entre los 7 y 10 días después de la siembra, la ramificación comienza entre los 45 y 50 días; la floración entre los 90 y 100 días y la madurez fisiológica se alcanza a partir de los 120 días dependiendo de las condiciones climáticas.

En los años 2017 y 2018 no se observaron plagas o enfermedades en las parcelas de los productores entrevistados.

\section{Talleres con la comunidad de Varvarco}

En el taller del mes de mayo de 2018 participaron 20 personas de la comunidad de Varvarco y alrededores. Esta actividad completó los datos sistematizados de las entrevistas con testimonios muy valiosos. Los asistentes reconocieron a GV y $\mathrm{HV}$ como los cuidadores de las semillas de quínoa en la zona y a ZF como una huertera muy experimentada que siempre cultivó quínoa.

Así mismo, expresaron que en la zona la quínoa puede denominarse 'quingüa', 'kinwa' o ‘quinua'. En ese contexto, coincidieron en que Chile es el origen de las semillas obtenidas por intercambios comerciales o trueque de productos de la ganadería local por semillas u otros bienes.

De igual modo, estuvieron de acuerdo en que se trata de un alimento muy bueno, rico y completo, tal como lo señaló una de las participantes que vivía junto a su madre y hermanas en las cuevas de Los Bolillos a 19km de Varvarco (36 48'35,93”'S, $70^{\circ} 41^{\prime 16,36 ”) ~ “ . . . l o s ~ a n ̃ o s ~ q u e ~ n o ~ h a b i ́ a ~}$ carne nos la pasábamos a pura quinua”. Este y otros testimonios similares dan cuenta de una práctica agrícola local y sostenida de utilización de la quínoa de al menos 90 años en el área de estudio, que contribuyó en épocas de carestía a mantener la segu- ridad alimentaria de los habitantes de esta región del norte neuquino.

Los asistentes coincidieron en que la forma más difundida de consumo en la zona es el 'mote'. Esta comida consiste en "hervir los granos durante 15 o 20 minutos y una vez fríos se agrega miel o azúcar a gusto” señaló doña ZF productora de diferentes tipos de quínoa en la actualidad. Algunos de los jóvenes presentes recordaron haberla comido en su niñez y comentaron desconocer los motivos por los cuales dejaron de hacerlo.

En relación a la situación actual del cultivo de quínoa indicaron que la falta de agua por la sequía de los últimos años los obligó a reducir la superficie de todos los cultivos. En el mismo sentido, afirmaron que la quínoa que producen en la huerta alcanza para “darse el gusto" una vez cada tanto y guardar semillas. También reconocieron que dada la baja producción actual de quínoa no sería posible ofrecerla en el mercado local o a los turistas. Sumado a esto, otros opinaron que habría que tener algún tipo de maquinaria para acondicionar los granos si se aumentara la producción.

En el cierre de esta instancia participativa, muchos asistentes mostraron interés por la diversidad de productos a base de quínoa que se presentaron y solicitaron una capacitación en preparación de recetas para el siguiente taller.

En el segundo taller realizado en Varvarco en diciembre de 2018 participaron 25 personas del área urbana y rural. En la primera parte del mismo, los integrantes del grupo de Promotores Culturales del norte neuquino, radicados en diferentes municipios del departamento Minas, mostraron objetos utilizados en el pasado para medir volumen, recetarios de comidas locales 
y videos de entrevistas a reconocidos pobladores. Los materiales disponibles en su cuenta de Facebook y sitio web You Tube fueron las herramientas utilizadas para difundir y poner en valor la cultura regional. En esta parte, los asistentes concluyeron que el conocimiento de los agricultores es inseparable del cultivo y por lo tanto semillas y productores son parte del patrimonio cultural de la zona. Además reconocieron que la ficha que resume el trabajo de investigación realizado es el documento que avala los saberes locales vinculados al cultivo de quínoa (Figura 7).

Para el cierre de esta instancia participativa la capacitadora de cocina regional NL presentó 3 recetas y mostró que es posible utilizar quínoa para todo tipo de comidas en reemplazo del arroz.

\section{DISCUSIÓN}

Debido a las circunstancias políticas y económicas de finales del siglo XIX y principios del XX, gran parte de los habitantes del norte de Neuquén proceden de regiones de Chile dedicadas a la producción agrícola que se abastecían de ganado argentino para el consumo y la exportación. Alrededor de 1940, a pesar de los controles aduaneros establecidos por ambos países en la década del 30 , los pequeños productores ganaderos del norte de Neuquén continuaron el comercio con el país vecino entregando su ganado y abasteciéndose de azúcar, granos, té, porotos y semillas entre otras mercancías. En Argentina, el cultivo en pequeñas parcelas para el autoconsumo, les permitió tener una dieta variada sin abandonar sus hábitos alimenticios (Bandieri 1993).
De acuerdo con Susana Bandieri esta parte de la cordillera de Neuquén estuvo más integrada al mercado chileno que al argentino debido, entre otras causas, a que el gobierno Argentino orientó su política productiva hacia las regiones Pampeana y Litoral. En tales circunstancias, el desarrollo del norte de la provincia se afectó y se empobrecieron los pequeños productores a causa de las sucesivas crisis económicas producto del cierre de la frontera con Chile, el fin de las actividades mineras, el traslado de la capital de Chos Malal a Neuquén entre otros factores vinculados al desarrollo del Estado nación (Silla 2010).

Al mismo tiempo, la proximidad geográfica con Chile fortaleció el vínculo cultural entre los pobladores en ambos lados de la frontera manifestada en tradición culinaria, la forma de hablar y de nombrar a los alimentos y las comidas de esta zona. En este sentido, Viola (2019) señala que la alimentación es parte de la herencia cultural que logró asentarse en el territorio traspasando las generaciones. Para los agricultores entrevistados en este estudio, en la actualidad el consumo de quínoa es para "darse un gusto", mientras que en el pasado fue para algunos "la comida del día". Sin embargo, se consumen los granos exclusivamente y no se utilizan las hojas como ocurre en el noroeste del país donde se preparan guisos, bocadillos y otras recetas (Costa Tártara et al. 2015).

En relación a las características generales del cultivo y ciclo vegetativo de la quínoa de Varvarco, si bien son necesarios más ensayos de campo para avanzar en el conocimiento de este recurso genético local, tienen muchas semejanzas con los materiales de la Macro Zona Centro de producción en Chile que limita con esta parte de Neuquén (Bazile et al. 2015, INIA 2018). 
QUINeA

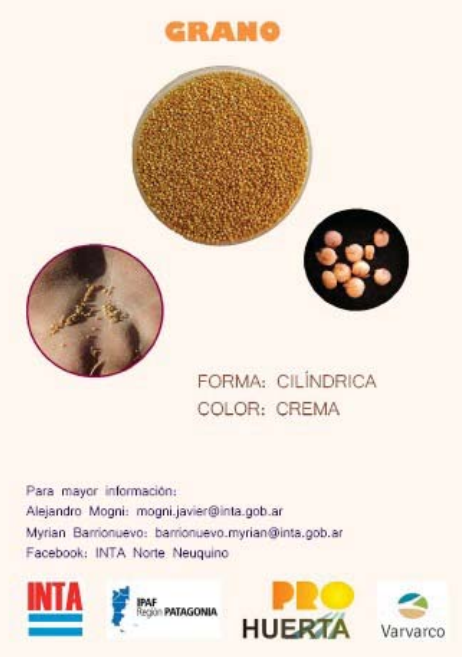

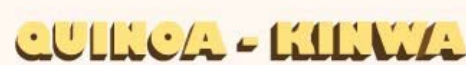

(Chenopedium quinea)

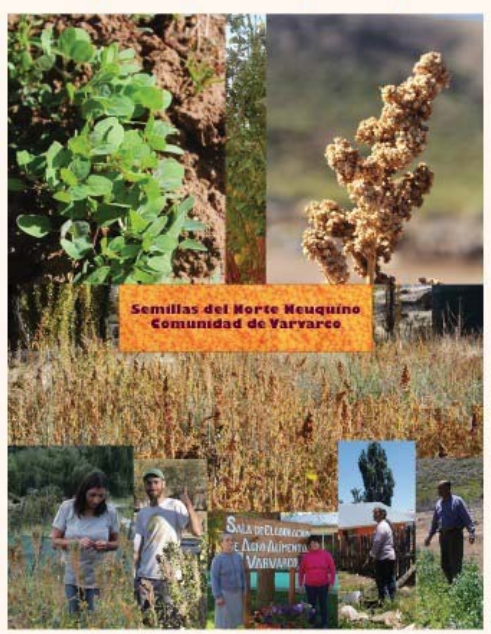

Nombre asignado por la comunidad de Varvarco en el Norte Neuquino: kinwa • quinua

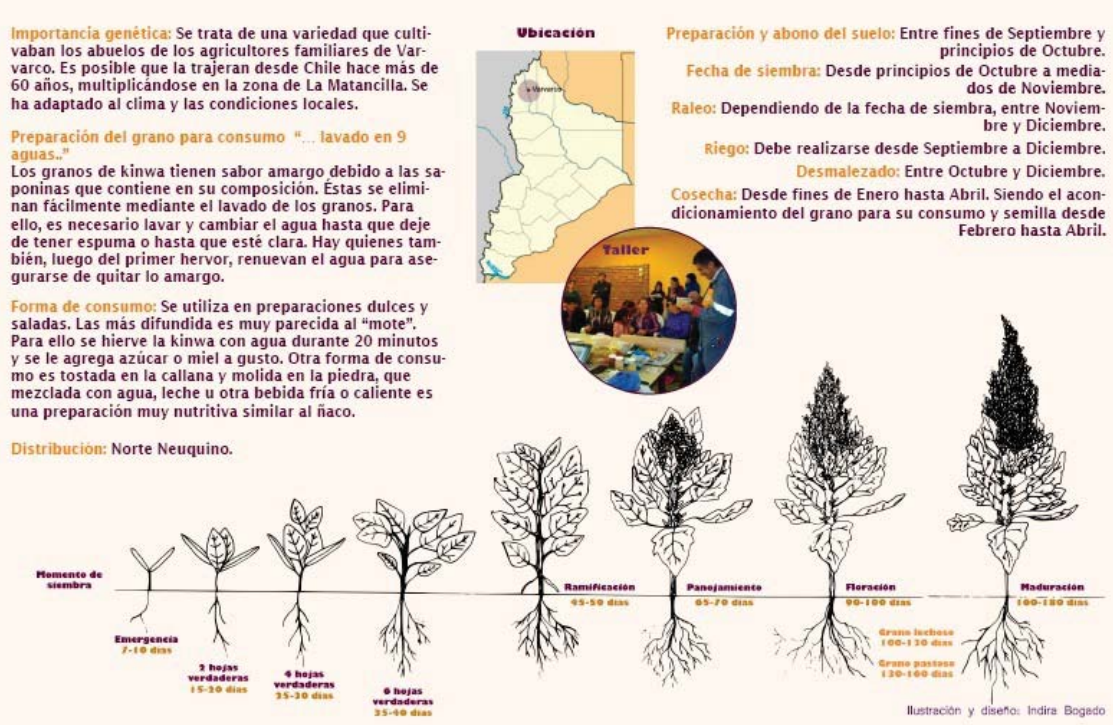

Figura 7. Ficha que resume el ciclo y principales características de la quínoa elaborada por el equipo técnico y la comunidad de Varvarco 2018.

Figure 7. Poster that summarizes the cycle and main characteristics of the quinoa prepared by the team and the community of Varvarco 2018. 
Además, los criterios de manejo de la diversidad de quínoa en el norte neuquino son similares a los descriptos por Bécares y Bazile (2009) para el centro de Chile (regiones de Maule y O’Higgins), donde los agricultores cultivan una variedad de quínoa -rara vez 2-; cuyas semillas son por lo general transmitidas por la familia de generación en generación y que consiguen de los vecinos solo en caso de pérdida. Por lo expuesto, es probable que la quínoa de Varvarco sea diferente a la quínoa Mapuche o 'dawe' cultivada en Ruca Choroy (departamento Aluminé), ya que las mujeres mantienen los intercambios de semillas con las comunidades mapuche de la VIII Región de Chile. En esa región, el cultivo es básicamente realizado en pequeñas superficies, con enfoque de agro biodiversidad y con características diferentes a las del centro y norte de Chile (Bazile et al. 2015). En virtud de este conocimiento sería conveniente realizar pruebas con todas los materiales presentes en la cordillera neuquina a fin de conocer el comportamiento agronómico y un posible aumento de escala en la producción además de mantenerlos vivos en las comunidades.

Por otra parte, a partir de los testimonios es posible reconocer algunas limitantes para el desarrollo del cultivo actual y potencial de quínoa en el norte neuquino. Unas de tipo ecológicas como la sequía que afecta a la región producto del cambio climático (CIMA 2015, Camilloni 2018) y otras de índole social debido al envejecimiento de los agricultores familiares y las migraciones desde las áreas rurales hacia los pueblos en busca de mejores oportunidades económicas y de acceso a vivienda, salud y educación de las familias productoras.
Sumado a lo anterior, para mantener y aumentar la escala de producción es necesario contar con maquinaria para la trilla y el escarificado de los granos. En este punto, cabe mencionar que los cultivos agrícolas en esta región son -mayormente- parte del sistema ganadero trashumante, que se complementa con otras actividades como son el empleo público o privado y los emprendimientos turísticos, entre otros (Bendini et al. 2004). En consecuencia, se debería contemplar las estrategias y los conocimientos de los agricultores que con sus decisiones resguardan los recursos genéticos a la hora de promover el aumento de escala o la introducción de germoplasma.

De este modo, y dado el carácter complejo de la producción de alimentos, es necesario un análisis multidimensional que exceda el campo ecológico y que permita entender -en el actual contexto económico y social- el recorrido de la quínoa del norte neuquino, para brindar además elementos técnicos que sean de utilidad en la toma de decisiones y fortalezcan estos sistemas productivos. 


\section{CONCLUSIONES}

A modo de cierre podemos afirmar que la quínoa de Varvarco es el resultado del trabajo de agricultores familiares en el proceso de adaptación a las condiciones ecológicas y los acontecimientos históricos y sociales que afectaron a esta parte de la cordillera neuquina. Por este motivo la comunidad de Varvarco reconoce la labor de los guardianes de semillas en el abastecimiento de la región donde se cultiva en el presente.

Los testimonios de productores, pobladores y técnicos confirman el cultivo de quínoa por agricultores criollos del que no hay registro escrito. De ahí que las metodologías participativas facilitan los procesos colectivos de intercambio de conocimiento, la valoración de saberes locales y la caracterización in situ del material genético de manera comunitaria.

Así, este material con características fenotípicas y etapas fenológicas similares a las quínoas del centro de Chile contribuyó a la seguridad alimentaria de las familias productoras en los periodos de escasez.

De este modo la información de base generada a partir del presente estudio mejora el conocimiento en relación al comportamiento de la quínoa local para adaptar los calendarios y densidades de siembra, el manejo del suelo, la sanidad y la cosecha a nuevas condiciones en otros agroecosistemas.

\section{AGRADECIMIENTOS}

A la comunidad de Varvarco por su generosidad y predisposición para compartir conocimientos, al proyecto INTA AUDEAS CONADEV CIAC N940167 “Caracterización y Conservación de Recursos Fitogenéticos Locales de la Agricultura Familiar en la Región Cuyo y Norpatagonia” y al Programa PRO HUERTA por el financiamiento para el desarrollo de las actividades.

\section{BIBLIOGRAFÍA}

Aucar A. 2004. Quínoa cultivo no tradicional... un camino hacia la sustentabilidad de las pequeñas comunidades. Neuquén: COPADE, Cdcyt.

Azar PF. 2002. Utilización de vegetales en las sociedades indígenas norpatagónicas: Contribución a una base de datos. [Tesis de grado]. [Buenos Aires]: Universidad Nacional de Buenos Aires.

Barrionuevo ME, Moronta NM, Mogni AJ, CabrapanV, Navarrete L. 2019. Conservación de la biodiversidad: sistema de semilla en el Norte Neuquino. [Disertación] [Cinco Saltos (Río Negro): Universidad Nacional del Comahue.

Bandieri S. 1993. Historia de Neuquén. Buenos Aires Editorial Plus Ultra. Capítulo 4.Actividades económicas y modalidades de asentamientos; p. 147-261.

Bazile D, Chia E, Olguín P. 2015. Diversidad de los cultivos de quínoa en Chile. Tierra Adentro. 108: 56-61. Disponible en: https:// agritrop.cirad.fr/578611/1/Bazile-Chia-Olguin_2015_TAD_Diversidad\%20de\%20 los\%20cultivos\%20de\%20quinoa\%20en\%20 Chile.pdf Consultada: 7 de abril de 2020. 
Bécares DA, Bazile D. 2009. La quínoa como parte de los sistemas agrícolas en chile: 3 regiones y 3 sistemas. Rev. geogr. Valpso. $\mathrm{N}^{\circ}$ 42: 61-72. Disponible en: http://agritrop.cirad.fr/554886/1/document_554886.pdf Consultada: 30 de marzo 2020

Bendini M, Tsakoumagkos P, Nogues C. 2004. Los crianceros trashumantes en Neuquén. Crianceros y Chacareros en la Patagonia. Cuaderno GESA. N 5, 23-39.

Bertero H D. 2001. Quínoa (Chenopodium quinoa, Chenopodiaceae) puede llegar a ser un cultivo importante para la agricultura argentina. Bol. Soc. Argent. Bot.36: 309-314.

Bioversity International, FAO, PROINPA, INIAF y FIDA. 2013. Descriptores para quínoa (Chenopodium quinoa Willd.) y sus parientes silvestres. Roma: Bioversity International y FAO. 52pp.

Camilloni I A. 2018. Argentina y el cambio climático. Ciencia e investigación. 68(5):5-10. Disponible en: https://ri.conicet.gov.ar/bitstream/handle/11336/99889/CONICET_Digital_Nro.cfeefb3b-550b-4172-bbdc-c39e534f7963_A.pdf?sequence $=2$ \&isAllowed $=\mathrm{y}$ Consultada: 15 de febrero de 2020.

[CIMA] Centro de Investigaciones del Mar y la Atmósfera. 2015. Cambio climático en Argentina; tendencias y proyecciones. Buenos Aires: CIMA. Capítulo 8, Cambios climáticos en la Región Patagonia, Antártida e Islas del Atlántico Sur; p. 280-341. Disponible en: http://3cn.cima.fcen.uba.ar/informe/ModClim_ Cap8.pdf Consultada: 15 de febrero de 2020.

Costa Tártara SM, Manifesto MM, Curti RN, Bertero HD. 2015. En Racionalidades campesinas en los Andes del Sur: reflexiones en torno al cultivo de la quinua y otros cultivos andinos. $1^{\mathrm{a}}$ ed. Salvador de Jujuy: Editorial de la Universidad Nacional de Jujuy. Capítulo 6, Origen, prácticas de cultivo, usos y diversidad genética de quinua del Noroeste Argentino (NOA) en el contexto del conocimiento actual del germoplasma de América del Sur; p. 199-230.
Costa Tártara SM, Manifesto MM, Bramardi SJ, Bertero HD. 2012. Genetic structure in cultivated quinoa (Chenopodium quinoa Willd.), a reflection of landscape structure in Northwest Argentina. Conserv. Genet. 13: 1027-1038.

Daza R, Burin D, Pereyra E, Heras AI. 2015. Quinua, regalo ancestral: historia, contexto, tecnología, políticas. Palpala (Jujuy): 128pp.

Gallardo A. 2019. Casa de semillas de uso comunitario. Experiencia del grupo de semillas de Zapala. Rev. Presencia 30 (72): 8-12.

Hunziker AT. 1943. Las especies alimenticias de Amaranthus y Chenopodium cultivadas por los indios de América. Rev. Arg. Agron. 30: 297-353.

[INDEC] Instituto Nacional de Estadísticas y Censo. 2010. Censo nacional de población, hogares y viviendas. 2010. Buenos Aires: INDEC.

[INIA] Instituto de Investigaciones Agropecuarias. 2018. Catálogo de variedades locales de quínoa: Zona Centro Sur de Chile. Santiago de Chile: INIA. 44pp. Disponible en: http:// www.inia.cl/recursosgeneticos/manual_quinoa.pdf Consultada. 15 de febrero de 2020

Knudsen H. 2000. Directorio de Colecciones de Germoplasma en América Latina y el Caribe. Roma: International Plant Genetic Resources Institute (IPGRI). 369pp.

Ladio A. 2005. Malezas exóticas comestibles y medicinales utilizadas en poblaciones del Noroeste patagónico: Aspectos etnobotáncios y ecológicos. Bol Latinoam Caribe Plant Med Aromat 4: 75-80. Disponible en: https:// www.redalyc.org/pdf/856/85640405.pdf Consultada: 3 de febrero 2020.

Lator C, Arias C, Pérez S. Las Ovejas, voces de identidad. $1^{\text {a }}$ Edición. Neuquén: Educo.

[LM] La Mañana Neuquén 2013. Edición en línea: http://www.treslineas.com.ar/la-manana-neuquen-f-112.html

| Revista FAVE - Ciencias Agrarias 20 (1) 2021 
Maloberti M, Korstanje MA, Quesada MN. 2016. Historizando la producción de quinua en el valle de El Bolsón (Departamento Belén, provincia de Catamarca). Mundo de antes. [En línea] 10: 117-141. Disponible en: https://ri.conicet.gov.ar/bitstream/handle/11336/56180/ CONICET_Digital_Nro.5ae90fa2-816b42bb-876c-750a3fe185a5_A.pdf?sequence $=2 \&$ isAllowed $=y$

Parodi LR. 1998. La agricultura aborigen argentina. Buenos Aires: Universidad de Buenos Aires. 48pp

Recalde J. 2007. Quínoa: posibilidades de su cultivo en las huertas familiares patagónicas. Boletín de Agricultura. 11:41-44

Silla R.2010. Variaciones temporales, espaciales y estacionales de los crianceros del norte neuquino. Revista Transporte y Territorio, 3: 5-22. Disponible en: www.rtt.filo.uba.ar/ RTT00302005.pdf

Viola M. 2019. Alimentos en tránsito. Tecnología y sistema agroalimentario. $1^{\mathrm{a}}$ ed. Lomas de Zamora: Universidad Nacional de Lomas de Zamora. 160pp.

Wilson HD. 1990. Quinoa and relatives (Chenopodium sect. Chenopodium subsect. Cellulata). Econ. Bot. 44: 92-110. 